\title{
Angiologie
}

\section{Neue Ansåtze zur Rezidivprophylaxe nach tiefer Venenthrombose?}

\author{
Tiefe Venenthrombosen (TVT) und in der Folge \\ Lungenembolien sind mit einer erheblichen Morbidität und \\ Mortalität behaftet. Ist es einmal zu einer TVT oder \\ Lungenembolie gekommen, ist das Rezidivrisiko hoch. Daher \\ sollte in diesem Fall über mindestens 3 Monate antikoaguliert \\ werden. Derzeit wird dafür im Allgemeinen ein Vitamin-K- \\ Antagonist, z.B. Warfarin, eingesetzt. Darunter sind jedoch \\ häufige Laborkontrollen notwendig, außerdem kommt es nicht \\ selten zu Blutungen. Zwei neue, oral einnehmbare Substanzen, \\ für die keine aufwendigen regelmäßigen Kontrollunter- \\ suchungen notwendig sind, werden daher aufmerksam \\ beobachtet. Nun gibt es aktuelle Studiendaten. \\ N Engl J Med 2013; 368: 699-708 und 709-718
}

\section{Apixaban: Die AMPLIFY-EXT-Studie}

Die randomisierte, kontrollierte Doppelblindstudie untersuchte den FaktorXa-Inhibitor Apixaban bei Patienten, die nach TVT oder Lungenembolie über mindestens 6 bis 12 Monate eine StandardAntikoagulation erhalten hatten. Danach sprachen ebenso viele Faktoren für wie gegen ein Fortführen dieser Behandlung. Insgesamt 2486 Teilnehmer wurden zwischen 2008 und 2011 aufgenommen und im Verhältnis 1:1:1 randomisiert $\mathrm{zu}$ Apixaban 2,5 mg zweimal täglich, Apixaban $5 \mathrm{mg}$ zweimal täglich oder Placebo für 1 Jahr. Der kombinierte primäre Wirksamkeitsendpunkt umfasste symptomatische Rezidive der venösen Thrombembolie und Tod jeder Ursache. Primärer Sicherheitsendpunkt waren schwere Blutungen (definiert als Abfall der Hämoglobinkonzentration um mindesten $2 \mathrm{~g} / \mathrm{dl}$, Transfusion von mindestens zwei Erythrozytenkonzentraten notwendig, Blutung an kritischer Lokalisation wie etwa intrazerebral, Blutung mit Todesfolge).

Ein Ereignis des primären Wirksamkeitsendpunkts erreichten innerhalb eines Jahres unter Placebo 11,6\%, unter Apixaban $2,5 \mathrm{mg} 3,8 \%$ und unter Apixaban $5 \mathrm{mg} \mathrm{4,2 \%}$ (für beide Vergleiche $\mathrm{p}<0,001$ ). Schwere Blutungen im Sinne des primären Sicherheitsendpunkts fanden sich bei
0,5\% unter Placebo, 0,2\% unter der 2,5mg-Dosis von Apixaban und 0,1\% unter der 5-mg-Dosis.

\section{Fazit}

Eine verlängerte Antikoagulation mit Apixaban reduzierte thrombembolische Rezidive; ohne eine erhöhte Blutungsrate nach sich zu ziehen. Allerdings waren die Studienteilnehmer relativ jung, und wenige hatten ein Körpergewicht unter $\mathbf{6 0}$ kg und eine moderate oder schwere Nierenschädigung. In diesen Gruppen müssten weitere Untersuchungen stattfinden, so die Autoren.

Sponsoring: Die Studie wurde von BristolMyers Squibb und Pfizer finanziert.

\section{Dabigatran: RE-MEDY- und RE-SONATE-Studie}

Zwei randomisierte Doppelblindstudien haben Dabigatran, einen direkten Thrombinhemmer, bei Patienten mit venöser Thrombembolie oder Lungenembolie nach mindestens 3-monatiger Standardbehandlung getestet. Dabei wurde in REMEDY gegen eine aktive Kontrolle (Warfarin) und in RE-SONATE gegen Placebo untersucht. Die Teilnehmer in RE-MEDY wiesen dabei ein erhöhtes Rezidivrisiko auf, sodass die Gabe von Placebo nicht vertretbar gewesen wäre. Insgesamt 2856 Patienten wurden in die aktiv-kontrollierte Studie aufgenommen, davon erhielten 1430 Dabigatran 150 mg zweimal täglich und 1426 Warfarin. An der Placebo-kontrollierten Untersuchung nahmen 1353 Patienten teil, und 681 wurden Dabigatran und 662 Placebo zugewiesen. In beiden Studien war der primäre Wirksamkeitsendpunkt ein gesichertes Rezidiv oder Tod durch Thrombembolie. Sicherheitsendpunkte umfassten schwere und klinisch relevante, nicht-schwere Blutungen.

In der aktiv-kontrollierten Studie kam es unter Warfarin bei 1,3\% zu einem thrombembolischen Rezidiv, unter Dabigatran bei $1,8 \%$ (Hazard Ration [HR] 1,44; 95\%Konfidenzintervall [KI] 0,78-2,64; p=0,01 für eine Nicht-Unterlegenheit von Dabigatran). Eine schwere Blutung trat in der Warfarin-Gruppe bei 1,8\%, in der Dabigatran-Gruppe bei $0,9 \%$ auf. Schwere oder klinisch relevante Blutungen waren unter Dabigatran seltener. Allerdings kam es unter Dabigatran häufiger zu akuten koronaren Ereignissen (0,9\% vs. $0,2 \%$ unter Warfarin; $\mathrm{p}=0,02$ ). Die Placebo-kontrollierte Studie zeigte Rezidive der Thrombembolie unter Dabigatran bei $0,4 \%$ und unter Placebo bei 5,6\% (HR 0,08, 95\%-KI 0,02-0,25; $\mathrm{p}<0,001)$. Schwere Blutungen traten bei zwei Patienten der Dabigatran$(0,3 \%)$ und bei keinem Patienten der Placebo-Gruppe auf. Schwere oder klinisch relevante Blutungen kamen bei 5,3\% unter Dabigatran und bei $1,8 \%$ unter Placebo vor (HR 2,92; 95\%-KI 0,52-5,60). Akute koronare Ereignisse fanden sich bei jeweils einem Patienten pro Gruppe.

\section{Fazit \\ Dabigatran in verlängerter Therapie kann thrombembolische Rezidive erfolgreich verhindern und führt zu einem geringeren Blutungsrisiko als Warfarin, so die Autoren. Der erhöh- ten Rate an koronaren Ereignissen gegenüber Warfarin müsse weiter nachgegangen werden.}

Sponsoring: Die Studie wurde von Boehringer Ingelheim finanziert.

Dr. med. Elke Ruchalla, Trossingen DOI 10.1055/s-0033-1338012 